\title{
Investigation of Electrical and Thermoelectric Properties of ZnO/rGO Composites Prepared by Conventional Solid-state Reaction Method
}

\author{
Suraj Mangavati ${ }^{1}$, Ashok Rao ${ }^{1, *}$, Dheeraj Devadiga ${ }^{2}$, M. Selvakumar ${ }^{2}$, Monika Saxena $^{3}$, G.S. Okram ${ }^{3}$ \\ ${ }^{1}$ Department of Physics, Manipal Institute of Technology, Manipal Academy of Higher Education, \\ Manipal 576104, Karnataka, India \\ ${ }^{2}$ Department of Chemistry, Manipal Institute of Technology, Manipal Academy of Higher Education, \\ Manipal 576104, Karnataka, India \\ ${ }^{3}$ UGC-DAE Consortium for Scientific Research, University Campus, Khandwa Road, 452017 Indore, India
}

(Received 12 January 2021; revised manuscript received 18 February 2021; published online 25 February 2021)

\begin{abstract}
$\mathrm{ZnO/rGO}$ composites are prepared by the conventional solid-state reaction method. Structural properties of prepared compounds were studied using XRD technique and it is observed that all the samples are crystallized in wurtzite structure. Electrical resistivity measurements were carried out using four probe method employing a closed cycle refrigerator. With addition of rGO, colossal reduction in the resistivity is observed which is about three orders of magnitude smaller than that of the pure sample of $\mathrm{ZnO}$. Room temperature Hall measurements were performed to estimate the bulk concentration and results show that addition of rGO into $\mathrm{ZnO}$ matrix enhances the number of charge carriers. Thermopower measurements were carried out using differential dc method and a large reduction in Seebeck coefficient is observed.
\end{abstract}

Keywords: Electrical resistivity, Mobility, Activation energy, Thermopower, Low temperature.

DOI: 10.21272/jnep.13(1).01026

PACS numbers: 61. 05.Cp, 62.23.Pq,

72. 15.Eb, 72.15.Jf

\section{INTRODUCTION}

The energy requirement to lead a better life is increasing during last few decades. The available fossil fuels like petrol, diesel and compressed natural gas are depleting and their enormous utilization is a cause for environmental pollution and global warming [1] Renewable energy sources are good candidates for an almost pollution free energy sources. To produce large amount of renewable energy is the need of the hour. In the last few decades thermoelectric (TE) devices have attracted the scientific community as these are ecofriendly, have ability to work over wide range of temperature and have long lasting life [2]. The efficiency of TE materials is calculated by a dimensionless quantity represented by ZT which is given by [3, 4],

$$
Z T=\frac{S^{2} \sigma T}{k},
$$

where, $S$ is Seebeck coefficient, $\sigma$ is electrical conductivity, $k$ is thermal conductivity and $T$ is temperature in Kelvin.

Among various TE materials, oxide TE materials are found to be of great importance due to their nontoxic nature, easy availability, and high stability over wide range of temperatures [5]. $\mathrm{ZnO}$ is one among various oxides which have been studied and results show that it is a promising material which can be used for thermo electric devices.

Pure $\mathrm{ZnO}$ is an $\mathrm{n}$ type semiconductor with band gap in the range $3.2-3.5 \mathrm{eV}$ [6]. Several attempts have been made to enhance TE properties of $\mathrm{ZnO}$ with the addition of different dopants and composites. Michitaka Ohtaki et al. synthesized $\mathrm{Al}$ doped $\mathrm{ZnO}$ and reported about three orders of magnitude increment in the electrical conductivity [7]. Kiryl V. et al. studied high temperature TE properties of Zirconium and Aluminum co-doped $\mathrm{ZnO}$ and reported 2.3 fold increase in figure of merit [8]. Priyanka Jood et al. doped heavy elements $\mathrm{Bi}$ and In in $\mathrm{ZnO}$ and observed ultra-low thermal conductivity and high powerfactor [9]. Composites of $\mathrm{ZnO}$ with other compounds also exhibit promising TE properties. Ning et al. fabricated composites of $\mathrm{ZnO} / \mathrm{Cu}_{2} \mathrm{SnSe}_{3}$ and the sample with 2.4 vol \% of $\mathrm{ZnO}$ has exhibited $\mathrm{ZT}$ value 1.3 times more than that of pure $\mathrm{Cu}_{2} \mathrm{SnSe}_{3}$ matrix [10]. Zhiwei Zhou et al. studied thermoelectric properties of $\mathrm{SnTe} / \mathrm{ZnO}$ composites and obtained highest ZT of 0.9 at $873 \mathrm{~K}$ for the sample with $x=0.8$ wt \% of $\mathrm{ZnO}$ which was $112 \%$ higher than that of the pure compound [11]. Most of the studies carried out in $\mathrm{ZnO}$ system are in high temperature regime. This motivated as to undertake low temperature studies on $\mathrm{ZnO}$ system. Here in this work, we have studied low temperature properties of $\mathrm{ZnO} / \mathrm{rGO}$ composites synthesized through cost effective solid-state reaction method.

\section{EXPERIMENTAL PROCEDURE}

The process for synthesis of graphene oxide (GO) is as follows. A 9:1 mixture of concentrated $\mathrm{H}_{2} \mathrm{SO}_{4}-\mathrm{H}_{3} \mathrm{PO}_{4}$ (360:40 mL) was added to a mixture of graphite flakes (3.0 g, 1 wt. equiv., SP-I Bay carbon) and $\mathrm{KMnO}_{4}$ (18.0 g, 6 wt. equiv.). The reactants were then heated to $50{ }^{\circ} \mathrm{C}$ and stirred for $12 \mathrm{~h}$. The reactants were cooled to room temperature and poured onto ice with $30 \% \mathrm{H}_{2} \mathrm{O}_{2}$ $(3 \mathrm{~mL}) .200 \mathrm{~mL}$ of water, $200 \mathrm{~mL}$ of $30 \% \mathrm{HCl}$ and $200 \mathrm{~mL}$ of ethanol (twice). The resultant suspensions

\footnotetext{
*ashokanu_rao@rediffmail.com

The results were presented at the International Conference on Multifunctional Nanomaterials (ICMN2020)
} 
were filtered. The filtered GO was dried under oven at $90^{\circ} \mathrm{C}$, which resulted in yellowish brown powder [12].

This was followed by synthesis of reduced graphene oxide (rGO). For preparing the rGO, $0.1 \mathrm{~g}$ GO was dispersed in $100 \mathrm{~mL}$ DI water for $90 \mathrm{~min}$. Then, $1 \mathrm{~mL}$ hydrazine hydrate was added to the prepared dispersion, and the mixture was heated to $95^{\circ} \mathrm{C}$ in an oil bath with a magnetic stirring for $24 \mathrm{~h}$ and finally, centrifuged and dried at $25^{\circ} \mathrm{C}$ [13].

$\mathrm{ZnO} / x$ wt $\%$ rGO ( $x=0,5$ and 10$)$ composites were prepared using the process as under. Pure $\mathrm{ZnO}$ powder (Merck, > $99 \%$ pure) was preheated in a Muffle furnace at $700{ }^{\circ} \mathrm{C}$ for 12 hours to remove moisture, if any. After naturally cooling, the powder was finely grinded for an hour and transferred to alumina boat to anneal at $1100{ }^{\circ} \mathrm{C}$ for 24 hours to enhance the crystallinity. The annealed $\mathrm{ZnO}$ powder and rGO prepared by (improved synthesis method) were weighed in stoichiometric ratio and mixed in an agate mortar and pestle to get homogeneity. The mixture was cold pressed into pellets and sintered in a furnace at $200^{\circ} \mathrm{C}$ for 24 hours. This process was repeated twice to get homogeneous samples.

The prepared samples were characterized by various techniques. Powder XRD method was adopted to confirm the crystal structure of the prepared compounds with Rigaku X-ray diffractometer using $\mathrm{Cu}-\mathrm{K} \propto$ radiation. The resistivity of prepared samples was measured in a closed cycle refrigerator equipped with Keithley source meters using standard dc four probe method [14]. The four wire contacts were made on the rectangular sample with the help of adhesive silver paste. Room temperature Hall effect measurement was performed using Ecopia HMS5500 with $0.5 T$ magnetic field to estimate the room temperature carrier concentration and mobility [15]. Thermopower is measured by adopting differential dc method [16].

\section{RESULTS AND DISCUSSIONS}

\subsection{Structural Studies (X-ray Diffraction)}

The powder XRD (X-ray diffraction) of the prepared samples was carried out in the range 20-80 degree $(2 \theta)$ and the obtained diffraction patters are shown in Fig. 1. All the samples crystallized in the hexagonal wurtzite structure which is consistent with the results in literatures [17-19]. With the addition of rGO into the $\mathrm{ZnO}$ matrix, there is a slight change in the crystal structure from the pure $\mathrm{ZnO}$. The sample with $5 \mathrm{wt} \%$ of rGO shows a low intense broad secondary peak around 25 degree and the intensity of the peak is enhanced for the sample with $x=10$ wt \% which is shown in Fig. 2.

The average crystallite size of the prepared compounds is calculated by Debye Scherrer formula which is given by [20,21],

$$
D=\frac{K \lambda}{\beta \cos \theta},
$$

where, $K$ is shape factor (0.9 for spherical geometry), $\beta$ is FWHM (Full Width at Half Maxima), $\theta$ is Bragg's angle. The average crystallite size of the presently investigated samples lies in the range 40 to $60 \mathrm{~nm}$.

\subsection{Electrical Properties}

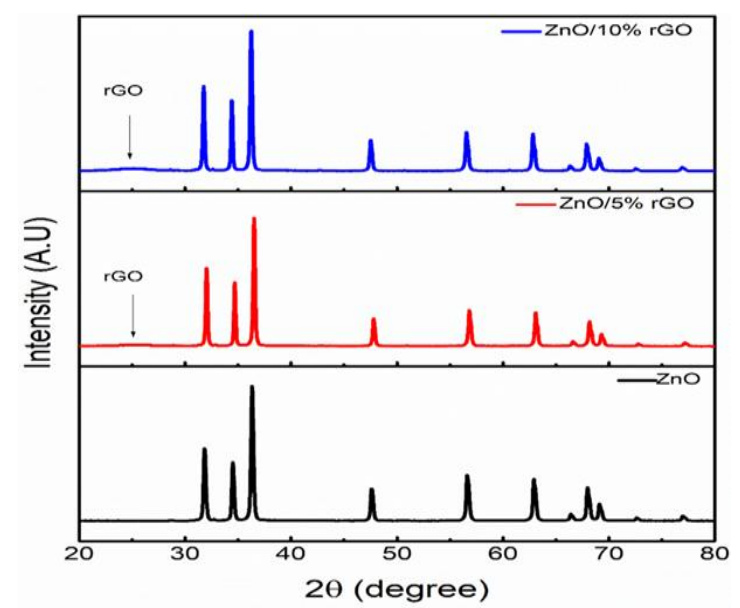

Fig. 1-XRD patterns of $\mathrm{ZnO} / \mathrm{rGO}$ composite samples composites

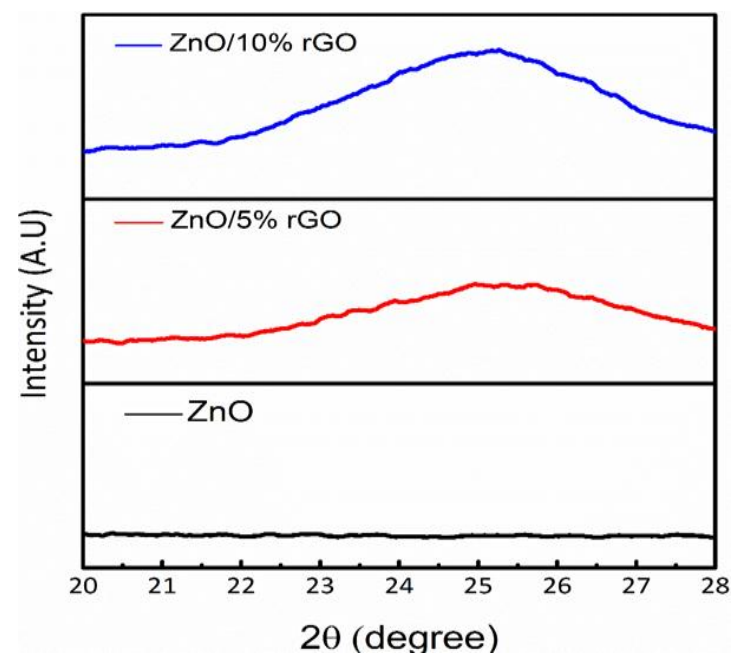

Fig. 2-Effect of rGO on XRD patterns of $\mathrm{ZnO} / \mathrm{rGO}$ composites

Electrical resistivity of the synthesized samples was measured in the temperature range $80-350 \mathrm{~K}$ and the plots are shown in Fig. 3. Resistivity of all the synthesized samples decreases with increase in temperature which indicates that the samples possess semiconducting behavior. Pure $\mathrm{ZnO}$ sample shows high resistivity of the order of $10 \mathrm{\Omega m}$. Addition of $5 \mathrm{wt} \%$ rGO into the $\mathrm{ZnO}$ matrix intensely reduced the resistivity to $10^{-2} \Omega \mathrm{m}$ and addition $10 \mathrm{wt} \% \mathrm{rGO}$ further reduced the resistivity to $10^{-3} \Omega \mathrm{m}$. The large reduction in the resistivity is attributed to the enhanced conductivity of rGO due to high carrier concentration. The bulk concentration is observed to be the maximum for the sample with $x=10 \mathrm{wt} \%$. On the contrary, mobility decreases with addition of rGO and the sample with $x=10 \mathrm{wt} \%$ shows minimum mobility. The room temperature carrier concentration, and mobility, are given in Table 1 . To understand the conduction mechanism, we adopted the Small Poloron Hopping (SPH) model which is given by [16],

$$
\rho=\rho_{0} T \exp \left(\frac{E_{A}}{k_{B} T}\right),
$$


where $\rho_{0}$ is a temperature independent constant, $E_{A}$ and $k_{B}$ corresponds to activation energy and Boltzmann constant respectively. The plot $\ln (\rho / T)$ vs $1 / T$ is shown in Fig. 4 and activation energy was calculated from the slope of the graph. The activation energies of pure and the composite samples are also listed in Table. 1 where it is observed that the activation energy decreases with addition of composites.

Table 1 -Room temperature bulk concentration, mobility, and activation energy of $\mathrm{ZnO} / \mathrm{rGO}$ samples

\begin{tabular}{|c|c|c|c|}
\hline Sample & $\begin{array}{c}\text { Bulk } \\
\text { concentration } \\
\left(\mathrm{cm}^{-3}\right)\end{array}$ & $\begin{array}{c}\text { Mobility } \\
\left(\mathrm{cm}^{2} / \mathrm{Vs}\right)\end{array}$ & $\begin{array}{c}\text { Activation } \\
\text { energy }(\mathrm{meV})\end{array}$ \\
\hline $\mathrm{ZnO}$ & $2 \times 10^{15}$ & 610 & 60 \\
\hline $\mathrm{ZnO} / 5 \% \mathrm{rGO}$ & $5 \times 10^{17}$ & 2.51 & 28 \\
\hline $\mathrm{ZnO} / 10 \% \mathrm{rGO}$ & $5 \times 10^{19}$ & 0.082 & 31 \\
\hline
\end{tabular}

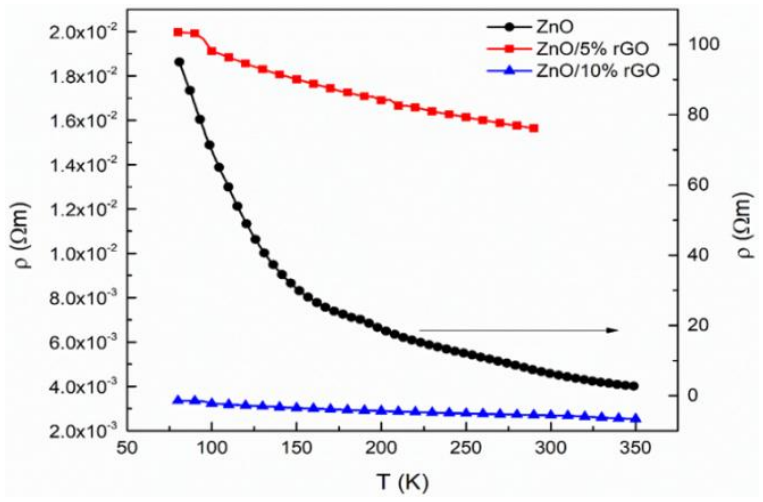

Fig. 3 -Temperature dependent resistivity plots of $\mathrm{ZnO} / \mathrm{rGO}$ composites

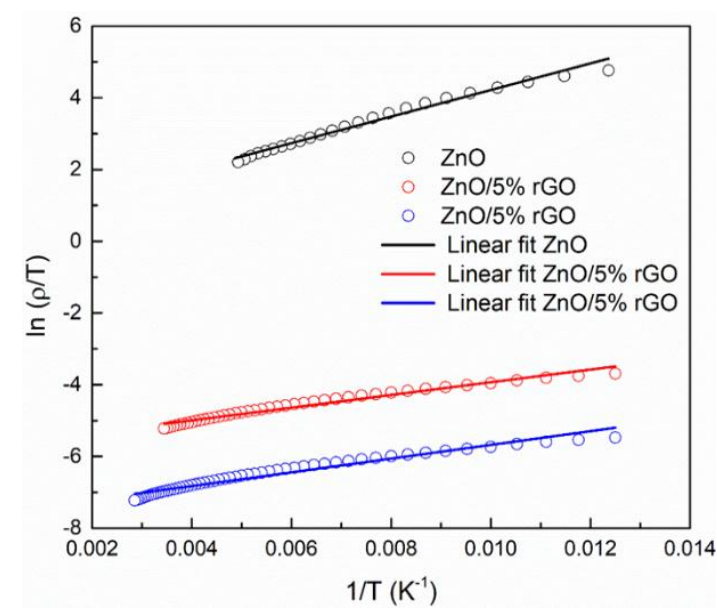

Fig. 4 - SPH fitting of $\mathrm{ZnO} / \mathrm{rGO}$ composites

\subsection{Seebeck Coefficient $(S)$}

Seebeck coefficient measurement of the samples was carried out in the temperature range $80-320 \mathrm{~K}$ and the variation of Seebeck coefficient with temperature is shown in Fig. 5. Due to high resistance of the pure sample, we could not measure the Seebeck coefficient at low temperatures. For the pure sample, the Seebeck coefficient first decreases with increase in the temperature, and then undergoes a change in sign around $190 \mathrm{~K}$ and thereafter it saturates around room temperature. The pure sample shows very high positive
$S$ value of around $23 \mathrm{mV} / \mathrm{K}$ at $160 \mathrm{~K}$ and it has a transition from positive $S$ to negative $S$ and reaches about $-15 \mathrm{mV} / \mathrm{K}$ around $190 \mathrm{~K}$. At room temperature, $\mathrm{S}$ remains negative with saturated value of $-1.4 \mathrm{mV} / \mathrm{K}$. Samples with $x=5 \mathrm{wt} \%$ also shows transition from negative to positive $S$ which could be attributed to asymmetric density of states near pseudogap [22] and increases linearly with temperature which indicates that the sample is degenerate semiconductor (heavily doped) [23]. The sample with $x=10 \mathrm{wt} \%$ shows positive Seebeck coefficient and also exhibit the degenerate semiconducting behavior. Addition of rGO into $\mathrm{ZnO}$ has reduced the $\mathrm{S}$ value by four orders of magnitude which could be attributed to enhanced carrier concentration of composite samples.

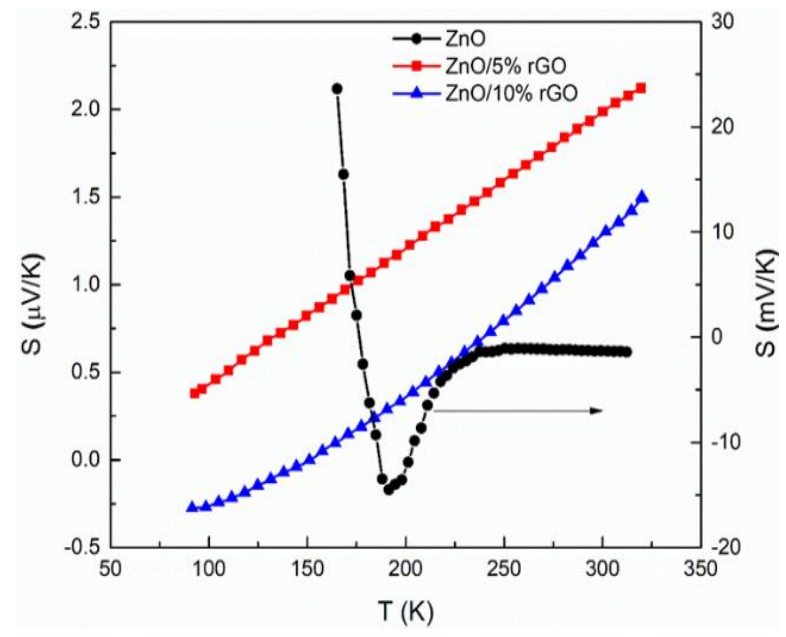

Fig. 5 - Temperature dependent Seebeck coefficient plots of $\mathrm{ZnO} / \mathrm{rGO}$ composites

\subsection{Power Factor (PF)}

Power factor is an important quantity in order to estimate the efficiency and output power of any TE material. In the recent past a study demonstrates that the strategy of lowering the thermal conductivity to enhance the efficiency of TE material can lead to thermo-mechanical problems whereas, tuning PF does not lead to thermo-mechanical instabilities [24].

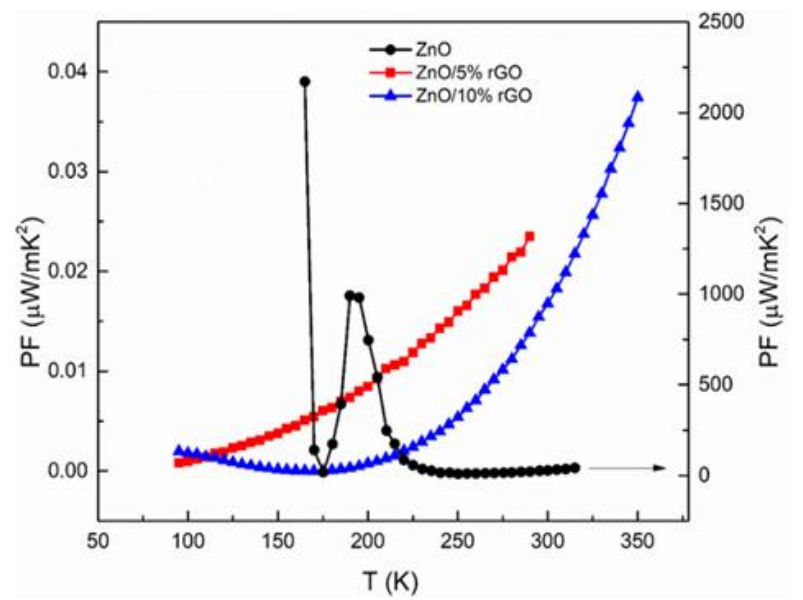

Fig. 6 - Temperature dependent power factor plots of $\mathrm{ZnO} / \mathrm{rGO}$ composites 
Therefore, we have estimated the power factor of $\mathrm{ZnO} / \mathrm{rGO}$ samples in the temperature range $80-320 \mathrm{~K}$ and Fig. 6 shows variation of $\mathrm{PF}$ with temperature. The highest $\mathrm{PF}$ is obtained for the pure sample $\left(\sim 2170 \mu \mathrm{W} / \mathrm{mK}^{2}\right)$ and at room temperature it reaches to $27 \mu \mathrm{W} / \mathrm{mK}^{2}$. With the addition of rGO into $\mathrm{ZnO}$ matrix, $\mathrm{PF}$ reduces to $0.02 \mu \mathrm{W} / \mathrm{mK}^{2}$ for the sample with $x=5 \mathrm{wt} \%$ and it further reduces to $0.01 \mu \mathrm{W} / \mathrm{mK}^{2}$ for the sample with $x=10 \mathrm{wt} \%$.

\section{CONCLUSIONS}

$\mathrm{ZnO} / \mathrm{rGO}$ composites were successfully prepared by solid-state reaction method. Prepared samples were characterized using various techniques. The effect of

\section{REFERENCES}

1. G.J. Snyder, E.S. Toberer, Nat. Mater. 7, 105 (2008).

2. X. Zhang, L.D. Zhao, J. Mater. 1, 92 (2015).

3. Lanling Zhao, Frank Yun Fei, Jun Wang, Funing Wang, Chunlei Wang, Jichao Li, Jiyang Wang, Zhenxiang Cheng, Shixue Dou, Xiaolin Wang, Sci. Rep. 7, 40436 (2017).

4. Jian Zhang, Xiaoying Qin, Di Li, Yongfei Liu, Yuanyue Li, Chunjun Song, Hongxing Xin, Xiaoguang Zhu, Appl. Phys. Lett. 108, 073902 (2016).

5. J. He, Y. Liu, R. Funahashi, J. Mater. Res. 26, 1762 (2011).

6. M. Ohtaki, J. Ceram. Soc. Jpn. 119, 770 (2011).

7. M. Ohtaki, T. Tsubota, K. Eguchi, H. Arai, J. Appl. Phys. 79, 1816 (1996).

8. Kiryl V. Zakharchuk, David M. Tobaldi, Xingxing Xiao, Wenjie Xie, Sergey M. Mikhalev, João F. Martins, Jorge R. Frade, Anke Weidenkaff, Andrei V. Kovalevsk, J. Eur. Ceram. Soc. 39,1222 (2019).

9. Priyanka Jood, Rutvik J. Mehta, Yanliang Zhang, Theo Borca-Tasciuc, Shi Xue Dou, David J. Singhd, Ganpati Ramanath, RSC Adv. 4, 6363 (2014).

10. J.A. Ning, D.G. Zhao, P. Jia, D. Wu, Mater. Sci. Forum 898, 1661 (2017).

11. J.Y. Zhiwei Zhou, Qinghui Jiang, Dan Zhang, Jiwu Xin, Xin Li, Yangyang Ren, 38, 42 (2016).

12. Y.N. Sudhakar, H. Hemant, S.S. Nitinkumar, P. Poornesh, M. Selvakumar, Ionics (Kiel). 23, 1267 (2017).

13. A. Mirmohseni, M. Azizi, M.S. Seyed Dorraji, Prog. Org. Coat. 131, 322 (2019). addition of rGO on the crystal structure was confirmed through X-ray diffraction studies by observing the broad secondary peak. A large reduction in the electrical resistivity was observed which is essential for a good thermoelectric material. A transition from negative to positive Seebeck coefficient is perhaps due to asymmetric density of states near pseudogap.

\section{ACKNOWLEDGEMENT}

The authors (SM and AR) are thankful to Department of Atomic Energy, Board of Research in Nuclear Sciences (DAE-BRNS), Government of India (2011/34/22/BRNS) and DST - FIST grant (SR/FIST/ PS-1/2017/8) for financially supporting this work.

14. R. Thomas, A. Rao, C.Y. Chung, Y.K. Kuo, B. Shivamurthy, Phys. B Condens. Matter 596, 412411 (2020).

15. K. Gurukrishna, A. Rao, Z.Z. Jiang, Y.K. Kuo, Intermetallics 122, 106803 (2020).

16. Y.R.S. Choudhary, Suraj Mangavati, Siddanagouda Patil, Ashok Rao, B.S. Nagaraja, Riya Thomas, G.S. Okram, Savitha G. Kini, J. Magn. Magn. Mater. 451, 110 (2018).

17. N. Lepot, M.K. Van Bael, H. Van den Rul, J. D'Haen, R. Peeters, D. Franco, J. Mullens, Mater. Lett. 61, 2624 (2007).

18. H. Colder, E. Guilmeau, C. Harnois, S. Marinel, R. Retoux, E. Savary, J. Eur. Ceram. Soc. 31, 2957 (2011)

19. M. Ullah, W. Chunlei, W. Bin Su, A. Manan, A. S. Ahmad, A.U. Rehman, J. Mater. Sci. Mater. Electron. 30, 4813 (2019).

20. C. Kulsi, K. Kargupta, S. Ganguly, D. Banerjee, Curr. Appl. Phys. 17, 1609 (2017).

21. M. Ahmadipour, M.J. Abu, M.F. Ab Rahman, M.F. Ain, Z.A. Ahmad, Micro Nano Lett. 11, 147 (2016).

22. A. Sakai, S. Yotsuhashi, H. Adachi, F. Ishii, Y. Onose, Y. Tomioka, N. Nagaosa, Y. Tokura, 2007 26th International Conference on Thermoelectrics, 256 (2007).

23. Shyam Prasad K, Ashok Rao, Bhasker Gahtori, Sivaiah Bathula, Ajay Dhar, Chia-Chi Chang, Yung-Kang Kuo, Phys. B Condens. Matter 520, 7 (2017).

24. W. Liu, H.S. Kim, Q. Jie, Z. Ren, Scripta Mater. 111, 3 (2016). 